\title{
Reform and Innovation of Economics Experiment Course for Finance and Economics Application-Oriented Undergraduate
}

\author{
Yingji Luo, Jianhua Zhang \\ National Demonstration Center for Experimental Economics and Management Education, \\ Guangxi University of Finance and Economics, Nanning530003, China
}

Keywords: Applied; Economics; Experiment; Reform

\begin{abstract}
Economics is the core basic course of applied undergraduate course of finance and economics, it emphasizes the high integration of knowledge and skills. However, the teaching effect shows that students are difficult to learn and difficult to use. In this paper, we take the problem as the guidance, with the idea of "multi-dimension", through the reform of the type of the experiment project, the experiment environment, the experiment content and the experiment method, the teaching model of the combination of theory, practice and experiment is innovated, and finally, the training effect of the integration of "teaching-practice" is achieved.
\end{abstract}

\section{财经类应用型本科经济学实验课程改革及创新设想}

\author{
罗应机，张建华 \\ 广西财经学院经济与管理国家级实验教学示范中心, 广西南宁，530003
}

关键词：应用型；经济学；实验；改革

摘 要: 经济学是财经类应用型本科核心基础课, 强调知识与技能的高度融合, 但教学效果 呈现学生难学难用的背离状况。论文以问题为导向, 以“多维并举” 的研究思路, 通过对课 程实验项目类型、实验环境、实验内容和实验方法的改革，创新理论、实践、实验相结合的 课程教学模式, 达到 “教用行” 融合的培养效果。

中图分类号：F011; F091.3 文献标识码：A 文章编号：CCPLCU042

\section{1. 引言}

在财经类应用型本科院校中, 强调知识与技能的高度融合, 经济学是一门重要的基础理论 核心课, 但历来又是学生感到最困惑的课程之一。主要原因在于这是一门理论性很强的课程, 在学术规范上又偏重于用大量的数学语言来表现经济原理, 我们在教学法上, 以介绍性为主 的孤立化理论教学模式给学生授课, 导致学生对教学内容无法感知和认知, 造成教学效果不 明显, 虽然能使学生了解一些经济学的基本知识, 记一些公式、图形, 却不能达到培养能力 和提高素质的目的, 甚至造成一些学生认识上的模糊。为了发挥出经济学实验的应有作用, 必须对经济学实验进行改进与创新。使学生提高学习经济学的兴趣, 加深对经济学理论和现 象的理解, 逐步完善以培养学生辩证、发散、求异、创新的思维能力, 提高科学研究能力为 目标的理论、实践、实验相结合的教学模式。

\section{2. 改革和创新的内部机理}

为了保障经济学人才培养目标的实现, 很多高校作了研究和探讨, 其中最大的举措是在 
经济学理论教学中引入实验的方法, 但在现实操作过程中, 由于受多种因素的干扰与制约, 经济学实验存在着不少问题与缺陷, 往往很难达到预想的实验效果。目前我国高校经济学实 验存在的问题主要有以下几个方面：一是实验项目设置不科学。目前我国只有少数高校开 设模拟、体验型实验和综合设计、研究创新型实验课程, 大多数高校的经济学实验课程仅停 留在演示、验证型实验的层面, 不少还是流于形式。二是实验流程与步骤设计不严密。目 前我国许多高校的经济学实验流程与步骤设计往往只重结果不重过程, 普遍不重视实验前期 准备和后期分析评估。三是实验信息的失真与误判。实验主持者在设计实验方案时往往无 法排除个人偏好和主观猜测, 在实验过程中欺骗、暗示、误导实验参加者的情形时有发生。 实验参加者也容易受实验主持者主观影响而被动消极地参加实验, 或顾及与实验主持者的关 系而有意识地完成实验期望; 四是实验的激励方法不合理。由于受高校实际情况的制约, 在经济学实验过程中很难以真实的货币报酬作为支付手段, 这不仅影响实验参加者的积极 性, 还妨碍实验主持者对实验参加者在实验进程的真实反应进行观察。

\section{3. 改革和创新的理论依据}

传统经济学实证分析的框架是思想实验的框架, 具体体现在对一些看不见的因素如人的欲 求、制度、技术、资源禀赋等作一些假定, 然后用严格的逻辑将这些假定和现实中的人们的 行为或现象联系起来, 证明人的某种看不见的行为为真时, 则相应的看得见的现象就会发生。 这种思想实验的过程所获得的假说或者理论, 需要用能够观察到的数据或现象来证明其真 伪, 但行为假设和实地数据获取的局限使经济假说和理论很难得到验证。所以美国经济学家 萨缪尔森和诺德豪在《经济学》一书中认为: “一种发现经济法则的方法可能就是通过被控 制的实验。不幸的是, 经济学家不容易控制其他重要因素, 因而无法进行类似化学或者生物 学家所做的实验, 他们一般只能像天文学家或气象学家一样借助于观察的手段”。

但20世纪70年代以后, 经济学的主导理论体系发生了变化。一般均衡理论、产业组织理 论、社会选择理论和公共选择理论将经济研究的假设由抽象拉回现实, 行为理论的成熟和理 性预期理论的出现为实验技术的发展创造了条件。正是在这样的背景下, 实验逐渐进入经济 学研究领域, 实验经济学逐步形成并发展成为现代经济学最重要的分支学科之一。

\section{4. 改革和创新的设想}

改革的目的是让学生具有扎实的经济理论基础、牢固的专业知识、熟练的操作技能。扎 实的经济理论基础要求培养学生拥有基本的经济素养和经济学视觉, 通过扎实的基础理论学 习, 使学生能够透过纷繁复杂的经济表象把握经济运行的一般规律, 并能在原有的基础上进 行理论创新。培养学生牢固的专业知识, 必须根据经济理论和实践的变化有选择、有区别、 有重点地向学生传授必要的专业知识。具有熟练的操作技能, 就是培养熟练的业务操作技 能, 对每个实验项目的设计都是贯穿培养学生的操作技能入手, 学生通过实验, 增强了对有 关经济理论知识的理解, 更好地帮助学生掌握经济理论知识。因此, 改革和创新的重点在 于:

\section{1 实验类型改革和创新}

实验项目类型是实验的核心内容, 必须花大功夫开发与精选经济学实验项目。首先, 要根 据经济学科的进步和社会发展需要, 以提高学生实践能力和创新能力为宗旨, 把以就业为导 向, 培养应用型人才的教学目标落实到经济学实验中。其次, 要根据专业的不同特点和学生 学习的不同阶段, 把解释性实验、验证性实验与综合分析实验有机结合起来, 分步骤、分阶 段地实施。

\subsection{1 解释性实验}

经济解释性实验课程是指利用经济理论去解释经济现象的一种实验课程。这种实验课程是 
从现实生活中的经济现象出发, 去寻找经济理论依据, 说明经济现象存在的道理。比如一张 百元人民币掉在路上, 在没有人看护的情况下，估计用不了多久，就会被过路的人捡走。这 个现象至少可以用两个经济学原理去解释它: 一是用需求定理去解释, 因为对于每个人来说 都需要人民币进行消费, 而且当路人捡走人民币时也不用花费什么代价, 就可以使手上的人 民币数量大增, 所以他把人民币拿走了; 二是用稀缺资源原理也可以解释这个现象, 因为人 民币对于一般人来讲是属于稀缺资源, 多胜于少, 人人都想得到它, 而且所拥有的人民币越 多越好，所以在没有别人看护的情况下，第一个路过的人就会把它捡起来占为已有了。

根据教师多年来的教学实践, 教师在组织这类经济实验课时一般可以采取两种教学组织方 式: 一种是在课堂讲授过程中通过选择恰当的经济现象去寻找经济理论, 从而可以加深学生 对经济理论的认识; 另一种是教师在讲述经济理论原理之后, 列举出与原理有关的经济现象, 让学生进行讨论, 由学生自己去找出经济现象的答案, 这样不但可以提高学生对经济原理的 认识, 而且还可以培养学生提高分析问题和解决问题的能力。所以经济解释性实验课程通常 是与理论教学同步进行的, 因此也可以把它称之为同步实验课程。

\subsection{2 验证性实验}

经济验证性实验是指在事先设定控制实验条件的范围, 由学生去进行自主实验进行验证, 从而掌握解决问题的程序和规律, 并加以应用的一种实验。这类经济实验往往在学生学习了 经济理论原理之后, 通过老师事先设计实验项目和要求, 学生根据实验给予的条件展开实验, 最终找出答案, 来证实经济理论的真理, 并在此基础上加以推广应用。在组织教学时一般采 用 “设定——验证——应用” 的教学模式。比如我们现在开设的计量经济学实验就是验证性 实验的范畴。当然这种实验也可以在事先建立操作软件平台基础上进行, 如我们开发的生产 交易软件平台就是其中之一, 只要赋予实验控制条件, 学生就可以在平台上完成实验任务。 由于验证性实验往往需要花费一定的时间, 实验过程比较复杂, 经历的实验环节比较多, 综 合性比较强, 因此这类实验不能象解释性实验那样随堂同步进行, 而应当单独开设实验, 集 中一定的时间去开展实验。

\section{1.3 综合分析实验}

前述的经济解释性实验和经济验证性实验基本上都属于单项实验, 它一般只针对某项经济 理论开展的实验, 而为了培养学生的经济综合分析能力, 还必须要开设综合经济分析实验。 所谓综合经济分析实验, 是指就经济的某个行业或整个国家的经济发展情况进行综合分析, 提出分析报告和经济发展的建设性意见和建议的一种实验。如农业经济分析实验、工商企业 经济分析实验、服务业经济分析实验、宏观经济数据分析实验等都属于综合经济分析实验。 这些实验的开设一般是先由老师进行示范性讲授, 如介绍某项经济发展的现状, 讲授如何收 集和整理好经济分析资料, 如何撰写经济分析报告等的基础上, 然后向学生提供选题方向, 由学生选定题目后自己去收集资料, 去开展实验, 最后写出综合经济分析报告。这类实验由 于综合性强，花费的时间多，学生投入的精力大，因此宜于集中时间进行开课。

\section{2 实验环境改革和创新}

实验环境的改革要与时俱进, 实现信息技术与课程项目深度融合。实验环境主要包括纸笔 型实验环境和虚拟仿真实验环境, 从我国对经济学实验研究和发展的现状来看, 各大高校主 要以人工控制的纸笔型实验模式为主, 采用的教室或会议室, 并借助纸笔黑板等设备进行, 道具主要有扑克牌、卡片、硬币等。纸笔型实验环境相对成熟, 改革的重点是虚拟仿真实验 环境的设计, 以计算机化的实验室和计算机等工具可作为实验辅助场所和工具, 借助统计软 件用于汇总实验数据和对实验数据进行统计分析, 通过信息技术对一些成本高、难以在现实 中实现、假设前提多、理论高度抽象的实验项目虚拟化。

\section{3 实验内容改革和创新}


实验内容要让学生感同身受, 改革的方向是西方经济理论与中国经济现状的结合, 完成理 论与现实经济现象的修正。通过源于现实真实数据模拟实验, 对采集的中国现实经济运行数 据加以整理分析, 运用本课题开发的计算机技术及软件进行整理建模, 并加以分析预测, 提 出对策。如在经济学中就业与经济增长之间的数量关系, 通常遵循奥肯定律, 但在发展中国 家, 这一理论受到明显的挑战。为了加深对奥肯定律的认识, 提高对理论基础知识转换为实 际应用能力, 对奥肯定律进行适当的扩张, 可尝试用就业量替代失业率构建扩大的奥肯模型, 分析中国的就业与经济增长的数量关系。

\section{4 实验方法改革和创新}

经济学实验流程与步骤的设计是否严密是保证实验结果准确真实的关键环节。一个严谨的 经济学实验包括设计与准备、具体操作与实施、分析与评估等3 个流程, 每一个流程又包括 若干步骤与程序。因此, 在进行经济学实验流程与步骤设计时, 要把经济学实验的前、中、 后 3 个流程有机联系起来, 流程中每一个步骤的设计都要力求严密细致, 确保实验结果的准 确与真实。

在实验前, 实验主持者首先要进行实验流程与步骤的设计及相关准备, 具体包括: 根据不同实验内容设计不同的实验方案。包括实验的目的、规则要求, 实验内容、实验步骤、 程序规划，实验用软件、资料、道具，实验参加人员的分组、分工等。

(2) 实验材料与工 具的准备。如道具、计算机、买卖双方收益记录表、市场数据统计表等。（3）实验场地准 备。包括室内实验室或室外交易场所。（4）制作实验说明书和工作人员手册。（5）组织学 生阅读实验说明书及相关表格, 让学生熟悉实验流程、步骤和要求。

\section{5. 创新和改革的难点和解决思路}

\section{1 实验道具的问题}

实验项目所需要的场所, 如传统教室或会议室比较容易解决, 但实验道具如专用的扑克牌、 卡片等在市场上没有销售。需要实验教师做好充足的实验前准备, 借签国内外成熟高校的经 验, 如国内有些高校正在开发经济学沙盘实验等, 另外由于工作量大, 对于实验教师可以给 予适当的激励措施。

\section{2 实验成本的问题}

本课题实验项目被试对象是学生, 要达到好的实验效果, 老师希望学生能够扮演实验中的 角色, 而不是把现实的角色带入。但实验项目实验对象效用的变化主要来自报酬, 如果采用 真实的货币激励, 会使实验效果更加明显, 在实验过程中做出符合实验要求的真实意愿行为, 但存在成本过高的问题。如果采用实验成绩或其它的非物质手段激励, 又无法满足实验者行 为的单调性、显著性和占优性原则, 如希望给老师留下深刻的印象获得高分等, 会干扰其在 实验中应该具有的目的性。可见, 利用正式货币比课程成绩更具激励性, 拟通过学科建设经 费和实验教学示范中心的倾向性政策解决实验成本的问题。

\section{3 实验师资问题}

经济学实验的实验对象是学生, 学生是人, 是有限理性的, 当学生非理性出牌时必然造成 实验结果与经济理论的预测值产生很大的差异, 从而导致实验失败。因此, 经济学实验对教 师的要求很高, 首先要求教师按照实验科学的要求模拟实验环境、确定实验步骤, 严格实验 监控、防止学生行为异化; 一旦学生行为异化导致实验数据与预测值有较大差异时, 教师也 能做出合理的分析与解释, 最终引导学生得出正确的结论。因而, 开展经济学实验对教师来 讲, 是比传统的课堂教学更大的挑战, 需要老师本身对经济学理论实质有深度的理解, 同时 了解国外经济学科研的前沿。 


\section{6. 结论}

为了达到财经类应用型人才的培养目标, 应当正视经济学实验课程中存在的问题, 从构建 经济学人才素质和知识的需要出发加以改革和创新。其中实验项目类型改革是重点, 按才培 养要求精分为解释性实验、验证性实验与综合分析实验三大类，根据学生的不同层次和不同 专业因才施教; 实验环境要能满足实验内容的要求, 除了传统的纸笔型实验环境外, 充分考 虑信息化手段与教学的结合; 实验内容要注意把经济学理论中国化, 更多的以中国经济事实 来解释; 在实验方法选择上, 要追求程序的严谨和流程的科学; 对于经济学实验的改革和创 新, 要解决在实验道具、实验师资和实验成本等方面的限制, 充分给予实验教师更多的激励 和重视。改革和创新的目的将是把干巴巴的 “黑板经济学” 理论转化成有血有肉的生动的 “事 实”, 在加深学生对经济学理论的理解的同时, 极大地激发学生学习经济的兴趣, 保证经济 学教学质量。

\section{Acknowledgements}

基金项目：经济与管理国家级实验教学示范中心（广西财经学院）：全息过程管理视角下 经管实验室仪器设备效用提升研究（ＳF Z X 2017 B 06）

作者简介: 罗应机 (1976一), 男 (汉族), 广西玉林人, 广西财经学院副教授, 硕士研 究生, 主要从事经济学和管理学研究。Email:421895997@qq.com

\section{[参 考 文 献]}

[1] Vernon L. Smith.Theory and experiment: What are the questions? [J]. Journal of Economic Behavior and Organization, 2009 (1) : 33-36

[2] Gary Bolton.Testing models and internalizing context [J]. Journal of Economic Behavior and Organization . 2009 (1):77-80

[3] Herbert Gintis.Towards a renaissance of economic theory [J]. Journal of Economic Behavior and Organization . 2009 (1):113-116

[4] Charness Gary,Fehr Ernst.From the lab to the real world. Science. 2015 (6260):158-161

[5] JosJ.Valero-Mas, JoséM. Iñesta. Interactive user correction of automatically detected onsets: approach and evaluation [J]. EURASIP Journal on Audio, Speech, and Music Processing, 2017, 2017(1):45-49

[6] 丹尼尔·豪瑟,罗卫东, 范良聪等: 实验经济学的兴起、发展及其在中国的应用前景 $[\mathrm{J}]$. 浙江 大学学报(人文社会科学版)，2012(04):56-59

[7] 赵明霏:理论经济学的实验教学问题研究[J]. 高等财经教育研究, 2016(04):78-80

[8] 李军军, 黄茂兴: 课堂实验在理论经济学教学中的应用与创新[J].福建师范大学学报(自然科 学版), 2011(03):98-102

[9] 唐雪峰. 实验经济学研究方法探新[J], 经济评论. 2006(04):125-127

[10] 高鸿桢,林嘉永. 信息不对称资本市场的实验研究[J].经济研究,2005(02):58-62

[11] 任蹈.实验经济学与经济仿真[J]. 理论与当代, 2007(02):112-115

[12] 林嘉永. 论经济学实验的科学设计[J]. 财经科学,2003(06):78-82

[13] 孙畅. 经济学实验课程设计的探索性研究[J]. 实验科学与技术,014(04):153-158

[14] 周业安. 经济学中的实验室实验: 经济学迈向科学研究的关键一步?[J]. 南方经 济, 2014(08):174-177 
[15] 王力南.经济学课程实验教学方法探究[J]. 高等教育研究, 2007(04):45-48

[16] 涂晓今.经济学实验[M]. 北京: 经济科学出版社 , 2008

[17] 张耀辉.实验经济学教程[M]. 北京: 经济科学出版社, 2006

[18] 马旭东. 浅析高校开设实验经济学的意义与方法[J]. 内蒙古财经学院学报(综合 版)，2009(02):162-165

[19] 彭文平. 关于经济学本科实验经济学教学的思考[J]. 考试周刊, 2009(11):31-33

[20] 朱庆. 实验经济学:兴起、应用与意义[J]. 国际经济合作, 2002(11):142-145 